\title{
Recurrent late seroma after immediate breast reconstruction with latissimus dorsi musculocutaneous flap
}

\author{
Seong Hwan Bae ${ }^{1,2}$, Yong Woo Lee ${ }^{1}$, Su Bong Nam ${ }^{1}$, So Jeong Lee ${ }^{3}$, Heeseung Park ${ }^{4}$, \\ Taewoo Kang ${ }^{4}$ \\ ${ }^{1}$ Department of Plastic and Reconstructive Surgery, Pusan National University School of Medicine, Busan; ${ }^{2}$ Biomedical Research Institute, \\ Pusan National University Hospital, Busan; ${ }^{3}$ Department of Pathology, Pusan National University School of Medicine, Busan; ${ }^{4}$ Busan Cancer \\ Center, Pusan National University Hospital, Busan, Korea
}

The latissimus dorsi musculocutaneous flap (LDMCF) is widely used for breast reconstruction. However, it has the disadvantage of frequent seroma formation at the donor site, and late seroma has also been reported. The authors report histological findings after the surgical treatment of a late, repeatedly recurrent seroma at 10 years after breast reconstruction with LDMCF. In 2008, a 66-year-old female patient underwent immediate breast reconstruction with LDMCF. In 2015, a late seroma was found at the donor site. After aspiration and drainage, the seroma recurred again in 2018. Total surgical excision of the seroma was performed and bloody-appearing fluid was identified in the capsule. The excised tissue was biopsied. Histological examination revealed no evidence of blood in the fluid, and multinucleated giant cells with amorphous eosinophilic proteinaceous material were identified. The cyst was suggestive of chronic granulomatous inflammation. There was no recurrence at 8 months postoperatively. The patient described herein underwent surgical treatment of late seroma that recurred after immediate breast reconstruction with LDMCF, and histological findings were identified. These results may be helpful for other future studies regarding late seroma after breast reconstruction with LDMCF.

Keywords Mastectomy / Mammaplasty / Surgical flaps / Superficial back muscles / Seroma
Correspondence: Taewoo Kang Busan Cancer Center, Pusan National University Hospital, 179 Gudeok-ro, Seo-gu, Busan 49241, Korea

Tel: +82-51-240-7281

Fax: +82-51-240-7746

E-mail: taewoo.kang@pusan.ac.kr

This work was supported by a clinical research grant from Pusan National University Hospital in 2018.

This article was presented as a poster at PRS Korea 2018 on November 9-11, 2018, in Seoul, Korea.

Received: March 30, 2019 • Revised: August 27, $2019 \bullet$ Accepted: September 19, 2019

pISSN: 2234-6163 • elSSN: 2234-6171 • https://doi.org/10.5999/aps.2019.00402 • Arch Plast Surg 2020;47:267-271

\section{INTRODUCTION}

Breast reconstruction with the latissimus dorsi musculocutaneous flap (LDMCF) was introduced in 1970, and is now one of the most commonly performed breast reconstruction procedures [1]. The advantages of this method include excellent esthetic outcomes, a relatively low complication rate, and the abil- ity to secure oncological safety. Seroma at the flap donor site is the most common complication, with a reported incidence of up to $79 \%$, and no single solution exists to dramatically reduce its incidence $[2,3]$. Donor-site seromas can cause several problems, such as discomfort and delays in postoperative chemotherapy $[2,4]$. Multiple studies have investigated seroma formation at the donor site after immediate breast reconstruction us- 
ing LDMCF $[3,5,6]$. However, relatively few studies have explored late seroma formation at the LDMCF donor site, and case reports presenting histological findings of seroma are likewise rare. The authors experienced a very rare case of recurrent late seroma at 10 years after breast reconstruction using LDMCF. Curative surgical treatment was applied, and histological results were obtained. The authors describe this case, along with a review of the literature.

\section{CASE}

A 66-year-old female patient (height, $155 \mathrm{~cm}$; weight, $53 \mathrm{~kg}$ ) with breast cancer (left breast; pT1N2M0, stage IIIA) underwent modified radical mastectomy and breast reconstruction using LDMCF in March 2008. Drainage was removed on the 12th day after surgery and the first round of chemotherapy was administered on the 21 st day postoperatively. During hospitalization, there were no complications at the donor site or any other issues, and she was discharged on the 36th day after the operation. She received three more rounds of chemotherapy after discharge, and outpatient follow-up for the next 7 years was uneventful. In July 2015, 7 years after surgery, the patient complained of a palpable mass on her back, at the donor site, at the outpatient clinic of the Department of General Surgery. Seroma was diagnosed, aspiration and drainage were performed, and the symptoms were relieved. In March 2018, 10 years after surgery, symptoms of a palpable mass recurred at the donor site. Incision, drainage, and bacterial culture were performed at the Department of General Surgery. The drained discharge from the donor site revealed a serous fluid pattern, including blood clot-like material, and no bacteria were identified in the culture.

\section{Fig. 1. Preoperative view of the wound}

The wound was found at the donor site after repeated incision and drainage for recurrent late seroma. There was still a palpable seroma at the donor site.

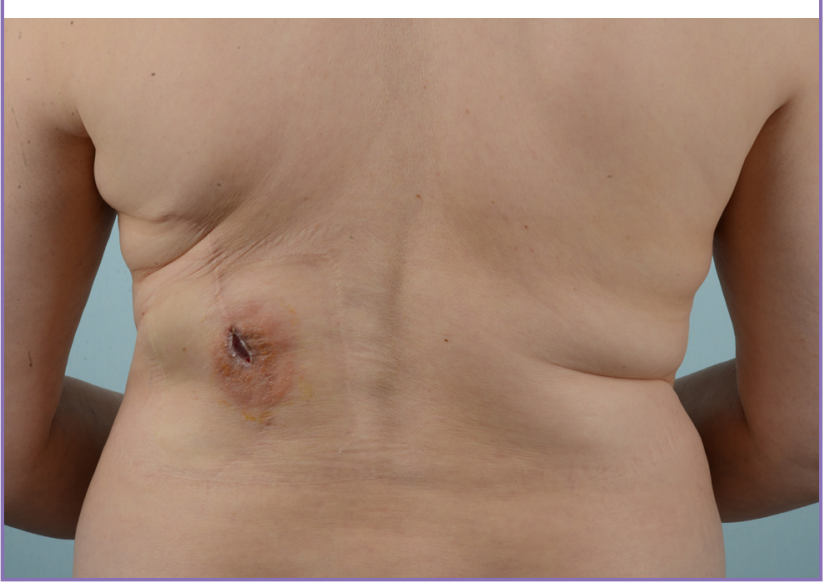

After repeated drainage of the serous fluid, the symptoms did not improve. Therefore, the patient was referred to the Department of Plastic Surgery, where she underwent irrigation and betadine gauze dressing (Fig. 1). In April 2018, the patient underwent surgery for the seroma under general anesthesia since serous discharge continued and the seroma remained unchanged. A serous capsule pouch located in the subcutaneous tissue layer was identified intraoperatively. The capsule pouch was completely excised by meticulous dissection and the inside of the capsule was observed. Within the capsule, serous fluid was found containing a brown-colored material with the appearance of a blood clot (Figs. 2-4). A permanent biopsy was performed of the completely excised serous capsule pouch. The histological findings showed that the brown-colored substance that had

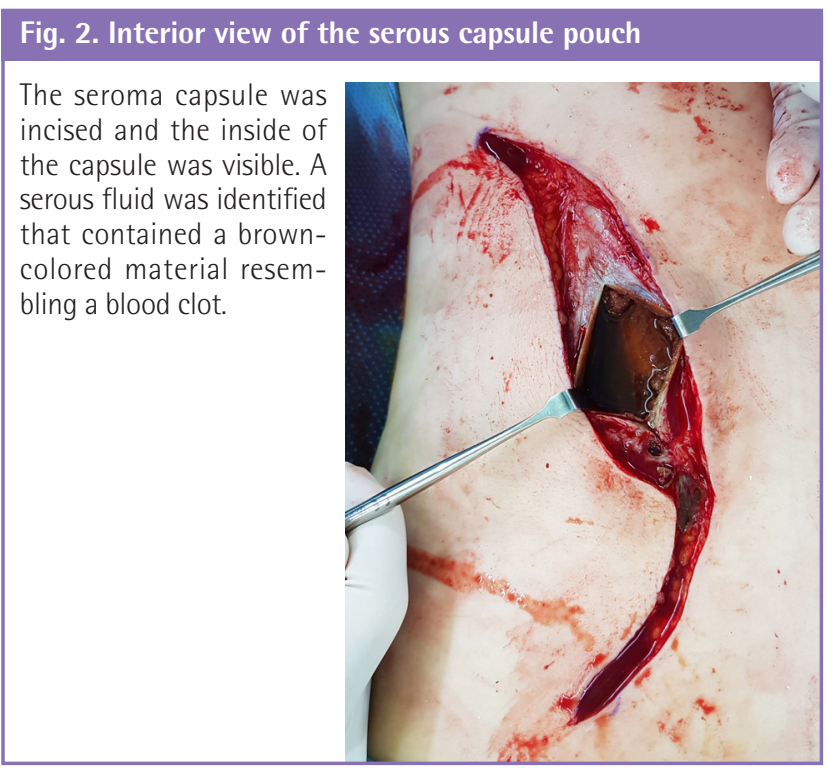

Fig. 3. Separation of the capsule pouch

Through meticulous dissection, the capsule pouch was separated from the surrounding tissue.

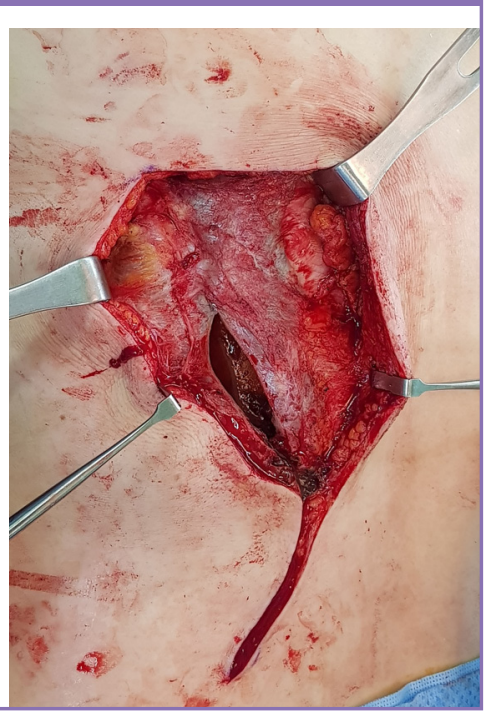


accumulated in the cyst was eosinophilic proteinaceous material, not a blood component. Multinucleated giant cells and epithelioid histocytes were irregularly distributed in the fibrous cystic wall that had formed around the brown-colored material. Therefore, the cyst was suggestive of chronic granulomatous inflammation (Fig. 5). Two weeks after the operation, the drainage volume was less than $30 \mathrm{~mL}$ and the drain was removed. The patient was then discharged. There was no evidence of seroma recurrence or other complications during 8 months of follow-up.

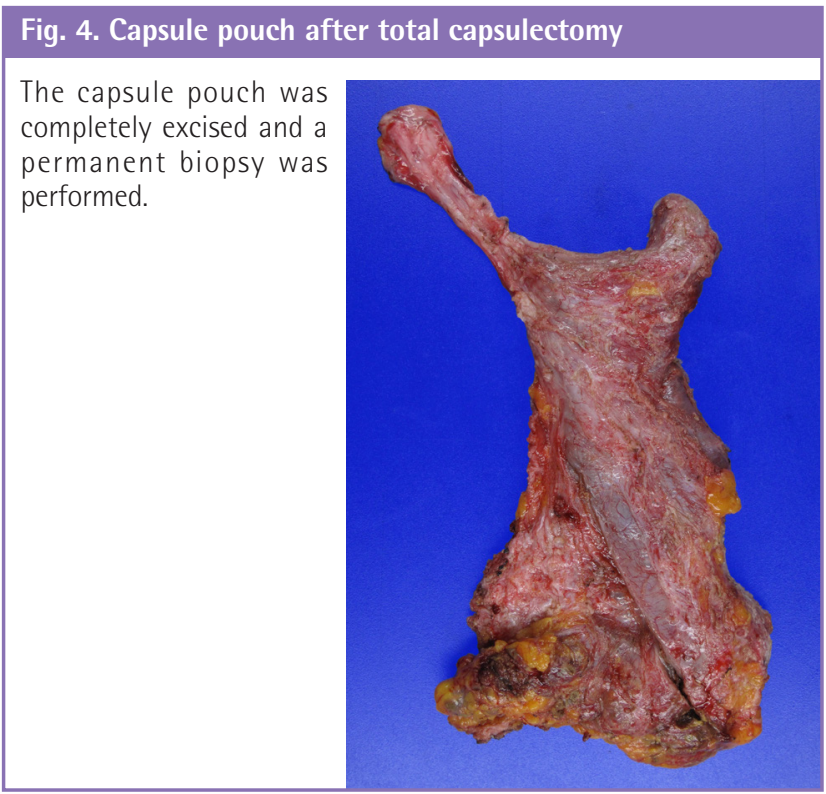

\section{DISCUSSION}

Donor-site seromas remain a noteworthy complication following LDMCF breast reconstruction. Many different methods have been tried to reduce the incidence of seroma formation. However, there is no known way to reliably reduce the risk of seroma formation, and systemic research is still lacking.

Various studies have described the following potential etiologies or factors contributing to seroma formation: advanced age, overweight, duration of keeping the drain in place, invasive breast surgery [5], nodal dissection [6], the extent of the mastectomy [7], and early shoulder mobilization [8]. Some researchers have suggested that seroma has an inflammatory origin, while others have proposed that seroma is likely to be derived from lymph $[9,10]$. In this case, most of the above-mentioned factors were not applicable or were difficult to identify. In addition, the histological findings included giant cells and histiocytes, suggesting the possibility of a chronic inflammatory origin, as well as being consistent with lymph $[11,12]$.

In our literature review, we found two previous studies on late seroma after breast cancer surgery. In 1998, Matsui et al. [13] reported the results of surgical resection and histological findings for a fibrous seroma capsule in the breast that occurred after mastectomy. In that study, the histological findings suggested that the capsule consisted of a seroma wall composed of collagen fibers with hyaline degeneration and a fibrous matrix containing lymphocytes and neutrophils. In 2007, Stanczyk et al. [14] reported a similar case, which ultimately required surgical resection of a fibrous encapsulated seroma after mastectomy

\section{Fig. 5. Microscopic view of the serous capsule pouch}

(A) An amorphous proteinaceous material was found as a cystic component. Multinucleated giant cells and epithelioid histocytes were irregularly distributed in the cystic wall $\left(H \& E_{1} \times 40\right)$. (B) Eosinophilic amorphous proteinaceous material and irregularly distributed multinucleated giant cells were present $\left(H \& E_{1} \times 200\right)$.
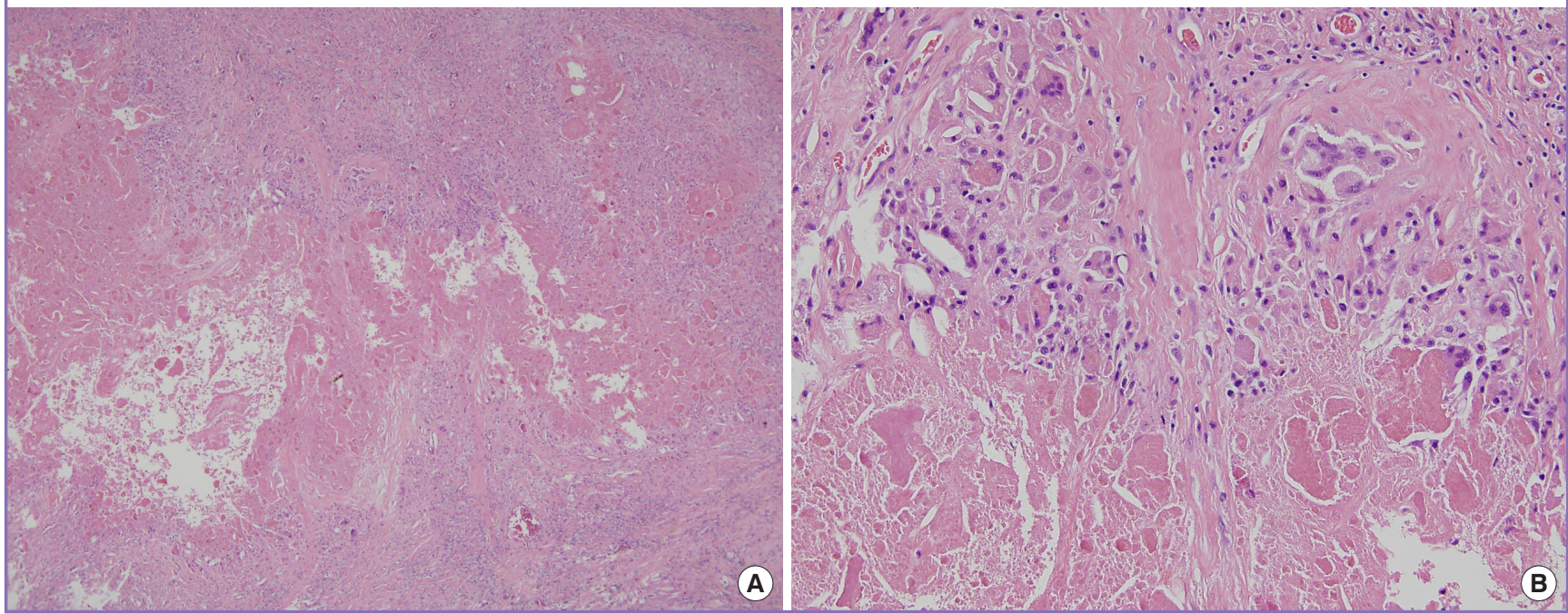
since conservative treatment was not effective. The authors of that study reported that the seroma capsule was mainly composed of lymphocyte-infiltrated fibrous tissue, with eosinophilic hyaline degeneration of collagen. A point of commonality between the case described in this report and the previous two studies is that surgical resection was performed for late seroma after mastectomy in a patient with breast cancer. In addition, the capsule pouch of the fibrous tissue wall was identified, and a histological examination was performed in all three cases. However, a comparison of the histological findings between this case and the previous ones indicates that there were some differences in the fiber and cell types constituting the seroma wall. The most significant difference is the location of the seroma. In the previous studies, the seroma occurred in the breast, whereas in this case, the seroma occurred at the donor site.

There are several limitations of this study that should be considered. One is that the components of the eosinophilic proteinaceous material that had accumulated in the cyst could not be identified more specifically based on the histological findings. Another limitation is that longer-term follow-up is needed due to the possibility of seroma recurrence after surgical resection of the seroma pouch. Meanwhile, a review of the medical history of the patient discussed in this study confirmed that a very small seroma was present at the donor site in chest computed tomography exams performed in 2012 and 2013. At that time, howev$\mathrm{er}$, the radiologists and surgeons were unaware of the presence of seroma. Although the seroma was very small, early detection and proper treatment would have helped to reduce the longterm risk of recurrence.

In conclusion, the authors successfully performed surgical excision to treat a recurrent late seroma at the donor site 10 years after mastectomy and breast reconstruction using LDMCF. A histological analysis was also conducted.

No other case studies have reported histological findings for a late serous capsule pouch that recurred repeatedly over a period of 10 years, although some cases have been reported of seroma at the donor site after mastectomy and breast reconstruction using LDMCF. Therefore, this study will help clinicians to recognize and manage such patients in the future.

\section{NOTES}

\section{Conflict of interest}

No potential conflict of interest relevant to this article was reported.

\section{Ethical approval}

This study was approved by the Institutional Review Board of
Pusan National University Hospital (IRB No. H-1908-003081) and performed in accordance with the principles of the Declaration of Helsinki. Written informed consent was obtained.

\section{Patient consent}

The patient provided written informed consent for the publication and the use of her images.

\section{Author contribution}

Conceptualization: SH Bae, YW Lee. Data curation: SB Nam, SJ Lee. Formal analysis: H Park. Methodology: SH Bae. Project administration: T Kang. Writing - original draft: YW Lee. Writing - review \& editing: SH Bae. Approval of final manuscript: all authors.

\section{ORCID}

Seong Hwan Bae https://orcid.org/0000-0002-7203-8978

Yong Woo Lee https://orcid.org/0000-0001-8088-822X

Su Bong Nam https://orcid.org/0000-0002-9661-0879

So Jeong Lee https://orcid.org/0000-0002-6465-9811

Heeseung Park https://orcid.org/0000-0001-9889-5217

Taewoo Kang https://orcid.org/0000-0002-6279-0904

\section{REFERENCES}

1. Malata CM, McIntosh SA, Purushotham AD. Immediate breast reconstruction after mastectomy for cancer. Br J Surg 2000;87:1455-72.

2. Menke H, Erkens M, Olbrisch RR. Evolving concepts in breast reconstruction with latissimus dorsi flaps: results and follow-up of 121 consecutive patients. Ann Plast Surg 2001; 47:107-14.

3. Schwabegger A, Ninkovic M, Brenner E, et al. Seroma as a common donor site morbidity after harvesting the latissimus dorsi flap: observations on cause and prevention. Ann Plast Surg 1997;38:594-7.

4. Clough KB, Louis-Sylvestre C, Fitoussi A, et al. Donor site sequelae after autologous breast reconstruction with an extended latissimus dorsi flap. Plast Reconstr Surg 2002;109: 1904-11.

5. Tomita K, Yano K, Masuoka T, et al. Postoperative seroma formation in breast reconstruction with latissimus dorsi flaps: a retrospective study of 174 consecutive cases. Ann Plast Surg 2007;59:149-51.

6. Randolph LC, Barone J, Angelats J, et al. Prediction of postoperative seroma after latissimus dorsi breast reconstruction. Plast Reconstr Surg 2005;116:1287-90. 
7. Say CC, Donegan W. A biostatistical evaluation of complications from mastectomy. Surg Gynecol Obstet 1974;138: 370-6.

8. Flew TJ. Wound drainage following radical mastectomy: the effect of restriction of shoulder movement. Br J Surg 1979; 66:302-5.

9. Watt-Boolsen S, Nielsen VB, Jensen J, et al. Postmastectomy seroma: a study of the nature and origin of seroma after mastectomy. Dan Med Bull 1989;36:487-9.

10. Bonnema J, Ligtenstein DA, Wiggers T, et al. The composition of serous fluid after axillary dissection. Eur J Surg 1999; 165:9-13.
11. Brodbeck WG, Anderson JM. Giant cell formation and function. Curr Opin Hematol 2009; 16:53-7.

12. Steinman RM. The dendritic cell system and its role in immunogenicity. Annu Rev Immunol 1991;9:271-96.

13. Matsui $Y$, Yanagida H, Yoshida $H$, et al. Seroma with fibrous capsule formation requiring a surgical resection after a modified radical mastectomy: report of a case. Surg Today 1998; 28:669-72.

14. Stanczyk M, Grala B, Zwierowicz T, et al. Surgical resection for persistent seroma, following modified radical mastectomy. World J Surg Oncol 2007;5:104. 